\title{
Evaluation of Soybean Resistance to Sclerotinia Stem Rot Using Reciprocal Grafting
}

T. D. Vuong, Research Associate, Department of Crop Sciences, University of Illinois; and G. L. Hartman, Research Plant Pathologist, USDA-ARS and Department of Crop Sciences, University of Illinois, 1101 W. Peabody Drive, Urbana, IL 61801

\begin{abstract}
Vuong, T. D., and Hartman, G. L. 2003. Evaluation of soybean resistance to Sclerotinia stem rot using reciprocal grafting. Plant Dis. 87:154-158.

Sclerotinia stem rot of soybean is one of the major soybean diseases in the north central region of the United States. One disease management option is to plant cultivars that have resistance. Some sources of partial resistance have been identified, but information pertaining to the nature of resistance is limited. The objective of this study was to determine if the expression of resistance is dictated by shoots of resistant plants and if this can be altered by using resistant and susceptible soybean genotypes grafted in different shoot and rootstock combinations of self-, single-, or double-shoot grafts. After successful grafts were made, several experiments were conducted using different inoculation techniques and soybean genotypes. In one experiment, cotyledons were inoculated with a plug of fungal mycelium, plants were incubated in a mist chamber for $23 \mathrm{~h}$, and plant survival was recorded over time. Based on seven grafting combinations of cross- and self-grafted plants using two soybean cultivars, grafts with NKS19-90 (partially resistant) as shoots had greater $(P \leq 0.05)$ plant survival at 3,4 , and 5 days after inoculation than the other graft combinations. In another experiment, a total of 17 graft combinations were generated using resistant plant introductions and two susceptible cultivars. Resistant selfgrafts of the plant introductions had greater $(P \leq 0.05)$ plant survival (mean $=75 \%)$ than selfgrafts of the susceptible cultivars (mean $=15 \%)$ at 5 days after inoculation. Inter-genotypic grafts with resistant shoots had greater $(P \leq 0.05)$ plant survival $($ mean $=65 \%)$ than those in reciprocal combinations (mean $=8 \%$ ) 5 days after inoculation. A cut stem inoculation method was used to test graft combinations of one resistant and two susceptible cultivars. Grafts with susceptible shoots of cvs. Williams 82 and Asgrow 2242 had greater $(P<0.05)$ lesion lengths $($ mean $=13.2 \mathrm{~cm})$ than shoots of NKS19-90 $($ mean $=9.2 \mathrm{~cm})$ regardless of the rootstock 15 days after inoculation. In a double-graft experiment, shoots of both NKS19-90 and Williams 82 were grafted to either NKS19-90 or Williams 82 rootstocks. Regardless of the rootstock, the shoots of Williams 82 died while shoots of NKS19-90 survived. For all the experiments, resistance was greater when the grafted shoot came from a resistant source on a susceptible rootstock compared with the reciprocal combination regardless of the type of grafting technique or inoculation method.
\end{abstract}

Sclerotinia stem rot of soybean is caused by Sclerotinia sclerotiorum (Lib.) de Bary. In Illinois, this disease was first reported in 1946 (5) and was considered a minor disease before 1990 based upon disease monitoring plots (8). However, during the last decade, the disease has been recognized as one of the most important diseases in Illi-

Corresponding author: G. L. Hartman

E-mail: ghartman@uiuc.edu

Trade and manufacturers names are necessary to report factually on available data; however, the USDA neither guarantees nor warrants the standard of the product, and the use of the name by USDA implies no approval of the product to the exclusion of others that may also be suitable.

Accepted for publication 10 September 2002.

Publication no. D-2002-1119-04R

This article is in the public domain and not copyrightable. It may be freely reprinted with customary crediting of the source. The American Phytopathological Society, 2003. nois, the north central region of the United States, and many soybean-producing countries in the world, where substantial yield losses have been reported (24).

In field environments, soybean plants initially are infected by $S$. sclerotiorum from ascospores that land on flowers (11). When moisture is adequate, they germinate and use the flower petals as a nutrient base. The fungus then infects and girdles the stem, often resulting in plant death. Typically, infected plants have necrotic leaves, bleached stem lesions, and white cottony mycelia on the plant surface when humidity is high. Sclerotia form inside and outside of infected tissues. Under controlled conditions in the greenhouse or growth chambers, disease symptoms may vary depending on the inoculation method, but in general infected stem tissue collapses and often appears bleached. With a cotyledon inoculation method (15), in which cotyledons of 2-week-old seedlings are inoculated with mycelial plug, first trifoliolates wilt and plants often die within 3 days. With a stem inoculation method (23), infected plants have typical bleached lesions on stems with sclerotia inside. There are few reports of basal stem infection of soybean; however, based on inoculation experiments by Chamberlain (5), both preand postemergence damping-off occur. There is no information on why root and basal infections are not as common in soybeans as they are in other crops. For example, in sunflowers, there are both head and root infections, and mycelial-germinating sclerotia were shown to cause root infection and wilt (13). Several inoculation systems were developed to assess resistance in sunflower to root infection based on basal stem symptoms (21). Additional observations and research are needed to determine if root and basal infection occurs in soybean under certain environmental conditions.

Some soybean genotypes have been reported to have partial resistance to $S$. sclerotiorum $(9,12,15,23)$. Under field conditions, both physiological resistance and/or escape mechanisms were reported to occur in soybean genotypes that had less disease $(4,12,19)$. Under greenhouse or laboratory experiments, most inoculation techniques evaluate physiological resistance. Although physiological resistance has been observed, there is little known about the mechanism of resistance to S. sclerotiorum; however, there is evidence that it is heritable (14). There is scant evidence of any genotype by isolate interaction, and it appears that the use of one aggressive isolate may be acceptable for tests conducted to evaluate resistance.

Grafting techniques have been used to study pathological and physiological responses in fruit and annual crop plants, including soybean. For example, grafting was used to study nitrogen fixation in soybean, and it was shown that soybean shoots regulated the rate of nitrogen fixation in the nodules (17). In other studies, supernodulating or hypernodulating soybean mutants grafted with wild-type parents indicated that isoflavonoids in shoots rather than roots were responsible for the inducement of hypernodulating mutation on roots $(6,7)$. For insect resistance, grafting was used to demonstrate that resistance factors from plant introductions were translocated to leaves throughout the plants of susceptible genotypes (16). Reciprocal grafts between brown stem rot resistant 
and susceptible soybean genotypes showed that resistance to brown stem rot was conditioned by roots and not shoots (2). Although Sclerotinia stem rot is primarily known as a stem disease of soybean, there is no indication whether disease resistance associated with stems is the result of stem and/or root response to infection. It is possible that under certain conditions, root or basal stem infection could occur by germination of sclerotia residing in the soil into infectious mycelia. The objective of this study was to determine if the expression of resistance is dictated by shoots of resistant plants and if this can be altered by using resistant and susceptible soybean genotypes grafted in different shoot and rootstock combinations of self-, single-, or double-shoot grafts.

\section{MATERIALS AND METHODS}

Plant growth conditions and inoculum preparation. Seeds were sown in a steamdisinfected soil:sand (1:1) mix in a greenhouse set at $27 \pm 2^{\circ} \mathrm{C}$ and 16 -h photoperiod with a photosynthetically active radiation of $434 \mu \mathrm{E} \cdot \mathrm{m}^{-2} \cdot \mathrm{s}^{-2} \quad$ (LI-170 Quantum/ Radiometer/Photometer, Lambda Instrument Corp., Lincoln, NE). At 5 to 6 days after sowing, seedlings were grafted and placed in a mist chamber at $100 \%$ relative humidity and $25 \pm 2^{\circ} \mathrm{C}$ for 6 to 7 days, then returned to the same greenhouse bench where seeds were sown and kept for 2 days before being transferred to another greenhouse room for inoculation. To inoculate plants, mycelial plugs $(0.5 \mathrm{~cm}$ in diameter $)$ were cut from the edges of 24-h-old colonies of $S$. sclerotiorum isolate $105 \mathrm{HT}$ (isolated from a soybean seed lot obtained from Story City, IA, in 1996 and stored as a stock culture on water agar at $4^{\circ} \mathrm{C}$ ). This isolate has consistently produced significant levels of infection through 6 years of use (G. L. Hartman, unpublished).

Cotyledon inoculation of single-shoot grafts of cultivars. Twenty seeds of three soybean cultivars, cv. NK S19-90, a partially resistant genotype (15), and cvs. Asgrow 2242 and Williams 82 (15), susceptible genotypes, were germinated in a 1:1 sand:soil mix in three separate rows in $26 \times 52 \mathrm{~cm}$ plastic trays. At 5 to 6 days after planting, half of the seedlings were reciprocally single-shoot grafted between resistant and susceptible genotypes; the remaining seedlings were self-grafted. For grafting, hypocotyls were cut $1 \mathrm{~cm}$ above the soil line and shoots were placed on the rootstock using a V-shaped graft (3). The grafts were held in place by $1-\mathrm{cm}$ sections of 3-mm-diameter plastic drinking straws. In this experiment, there were seven grafting combinations. Three were self-grafted plants; two were cv. NK S1990 as shoots with the two other cultivars as roots; and two were cv. NK S19-90 as rootstock with the two other cultivars as shoots. There were 7 to 10 plants per replication.
To inoculate plants, a single plug was placed mycelial side down on one cotyledon against the stem of each seedling. All seedlings and the insides of plastic domes were lightly atomized with water using a hand-held sprayer and covered. The domecovered trays were placed under black mesh cloth (80\% light reduction) at $20 \pm$ $2^{\circ} \mathrm{C}$ for 21 to $23 \mathrm{~h}$. The domes were removed and the trays were returned to the original greenhouse bench, where seeds were germinated for evaluation of disease response. The number of seedlings that survived was counted daily over 5 days, and the percentage of plants that survived was calculated. The experiment was a randomized complete block design (RCBD) with three replications, and it was performed twice.

Cotyledon inoculation of single-shoot grafts of PIs and cultivars. This experiment was as previously described for the single-shoot grafts of cultivars except that different genotypes were used. A total of 17 graft combinations were generated using resistant plant introductions PI549.076A, PI503.336, and PI194.639, and two susceptible cultivars, Alpha and Merit. There were 12 reciprocal grafts representing the grafts of the three PIs and the two cultivars and five self-grafts. The procedures of single-shoot grafting, cotyledon inoculation, and disease assessment were performed following the methods previously described. There were 7 to 10 plants per graft combination. The experiment was an RCBD with three replications. Values for the area under survival curve (AUSC) were calculated as follows: $\sum_{i=1}^{n-1}\left[\left(X_{i+1}+\right.\right.$ $\left.\left.X_{i}\right) / 2\right]\left[t_{i+1}-t_{i}\right]$, in which $X_{i}=$ percent survival at the $i$ th observation, $t_{i}=$ time (days after inoculation) at the $i$ th observation, and $n=$ total number of observations.

Cut stem inoculation of single-shoot grafts. Eight seeds each of NKS19-90, Asgrow 2242, and Williams 82 were germinated in 15 -cm clay pots. Five 6-day-old healthy seedlings were single-shoot grafted as previously described. There were seven graft combinations, which included three self-grafts, and NKS19-90 used either as rootstock or shoots for each of the cultivars. Each graft combination was performed in each pot. Grafted plants were incubated for 6 days in a mist chamber at $25 \pm 2^{\circ} \mathrm{C}$. The plants were transferred to an adjacent bench and allowed to grow for 4 weeks.

A cut stem inoculation method was used to evaluate resistance when the plants were 1 month old and the fourth trifoliolate leaf was fully expanded. Stems were severed with a razor blade $0.5 \mathrm{~cm}$ above either the third or fourth node. A mycelial plug as previously described was placed mycelial side down on the wound. Inoculated plants were incubated in a mist chamber at $20^{\circ} \mathrm{C}$ under high humidity for $48 \mathrm{~h}$. Infected plants were transferred to an adjacent bench. Lesion length of each stem was measured daily until day 15 after inoculation, and these data were used for analysis. There were five plants per treatment. The experiment was an RCBD with three replications, and it was performed twice.

Cut stem inoculation of double-shoot grafts of cultivars. In addition to the single-shoot technique, a double-shoot technique also was employed. Two shoots, NKS19-90 (R) and Williams 82 (S), were grafted to one rootstock, either NKS19-90 or Williams 82. Two to three grafts were made in each of two pots (replications) to make a total of five to seven double-shoot grafts for each combination. Self-grafts and plants that were not grafted were included. Incubation and inoculation procedures were performed as previously described. Disease symptoms including wilting and death of plants were observed daily for each graft and the controls until 5 days after inoculation.

Statistical analysis. An analysis of variance of the percent survival, AUSC values, and lesion lengths was completed using the general linear model procedure (PROC

Table 1. Analyses of variance for two grafting experiments of soybean inoculated with Sclerotinia sclerotiorum $^{\mathrm{a}}$

\begin{tabular}{|c|c|c|c|c|}
\hline Source of variation ${ }^{b}$ & df & MS & $\boldsymbol{F}$ & $P>F$ \\
\hline \multicolumn{5}{|l|}{ Cotyledon inoculation } \\
\hline Trial & 1 & 1,122 & 3.66 & 0.065 \\
\hline Replication (trial) & 4 & 303 & 0.99 & 0.428 \\
\hline Combination & 6 & 7,854 & 25.65 & $<0.0001$ \\
\hline Trial $\times$ combination & 6 & 165 & 0.54 & 0.775 \\
\hline \multicolumn{5}{|l|}{ Cut stem inoculation } \\
\hline Trial & 1 & 0.24 & 0.10 & 0.759 \\
\hline Replication (trial) & 4 & 2.23 & 0.90 & 0.479 \\
\hline Combination & 6 & 31.25 & 12.58 & $<0.0001$ \\
\hline Trial $\times$ combination & 6 & 0.27 & 0.11 & 0.994 \\
\hline
\end{tabular}

a The cotyledon inoculation experiment is based on plant survival 5 days after inoculation (DAI) and the cut stem inoculation methods is based on lesion lengths measured $15 \mathrm{DAI}$.

b Cv. NK S19-90, a partially resistant genotype (15), and cvs. Asgrow 2242 and Williams 82, susceptible genotypes (15), were reciprocally single-shoot grafted between resistant and susceptible genotypes, self-grafted, or not grafted. There were seven grafting combinations. Three were self-grafted plants; two were with cv. NK S19-90 as shoots with the two other cultivars as roots, and two were cv. NK S19-90 as rootstock with the two other cultivars as shoots. There were 7 to 10 plants per replication. Data from noninoculated controls were not used in the analysis. 


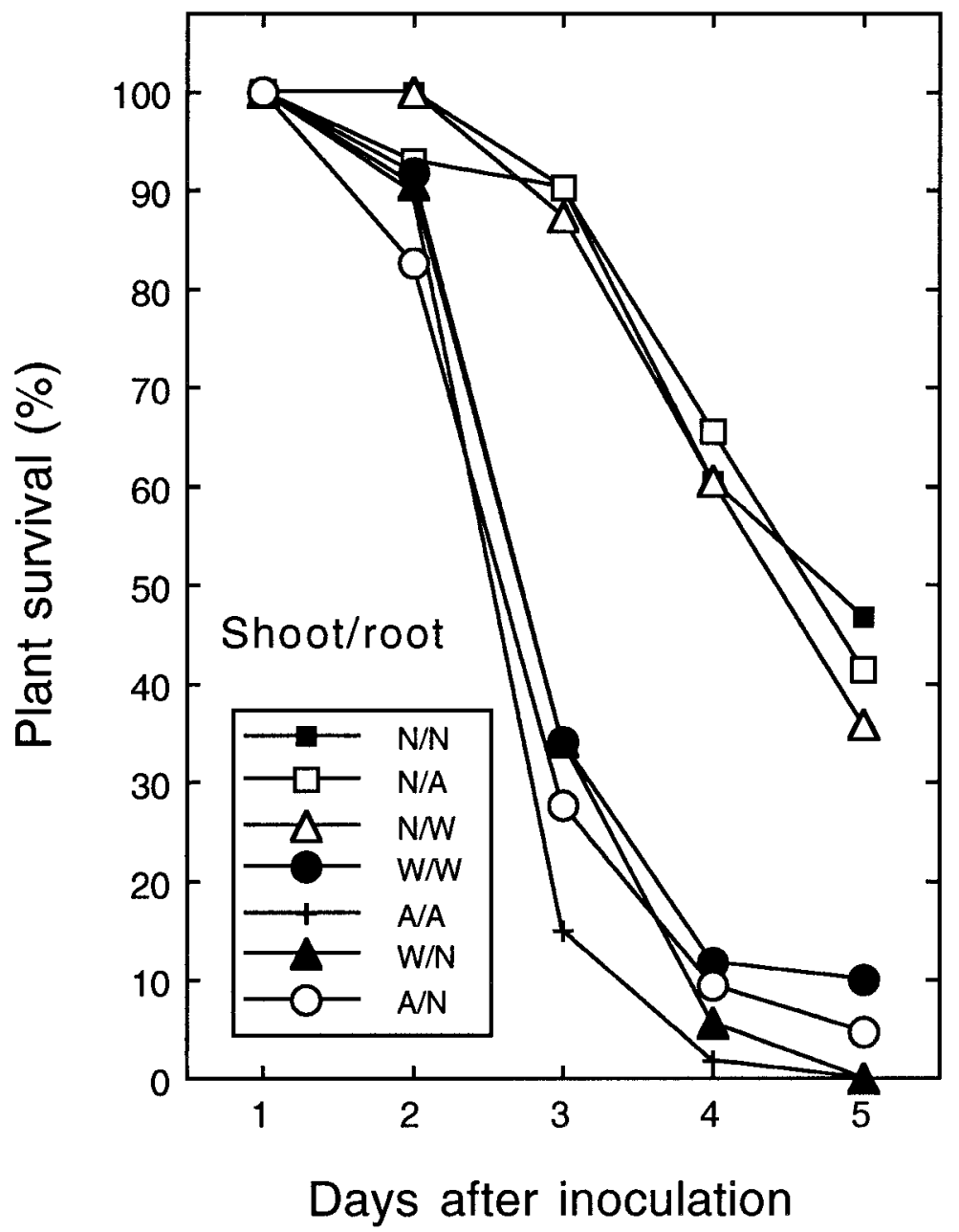

Fig. 1. Survival of soybean plants (\%) in cross- and self-grafting combinations among resistant and susceptible soybean genotypes 1 through 5 days after being inoculated with Sclerotinia sclerotiorum. $\mathrm{N}=$ NKS19-90 (resistant); A = Asgrow 2242 (susceptible); and W = Williams 82 (susceptible). Means are based on five plants per graft combination, three replications, and two trials. Least significant difference $(0.05)=19,23$, and 23 for 3,4 , and 5 days, respectively.

Table 2. Mean percent survival and area under the survival curve (AUSC) of soybean plants in crossand self-grafting combinations using partially resistant (PI549.076A, PI503.336, and PI194.639) and susceptible (Alpha and Merit) soybean genotypes inoculated with Sclerotinia sclerotiorum 1 through 5 days after inoculation (DAI)

\begin{tabular}{|c|c|c|c|c|c|}
\hline \multirow{2}{*}{$\begin{array}{l}\text { Graft combination } \\
\text { (shoot/root) }\end{array}$} & \multicolumn{4}{|c|}{ Percent plant survival $^{\mathrm{a}}$} & \multirow[b]{2}{*}{$\mathbf{A U S C}^{\mathrm{b}}$} \\
\hline & 2 & 3 & 4 & 5 & \\
\hline Alpha/Alpha & 51 & 16 & 9 & 9 & 131 \\
\hline Merit/PI194.639 & 43 & 40 & 15 & 7 & 152 \\
\hline Merit/PI503.336 & 50 & 42 & 14 & 7 & 159 \\
\hline Merit/Merit & 53 & 30 & 20 & 20 & 163 \\
\hline Merit/PI549.076A & 66 & 66 & 20 & 10 & 208 \\
\hline Alpha/PI549.076A & 83 & 66 & 33 & 8 & 236 \\
\hline Alpha/PI194.639 & 87 & 62 & 40 & 5 & 242 \\
\hline Alpha/PI503.336 & 100 & 66 & 33 & 0 & 249 \\
\hline PI549.076A/Merit & 75 & 75 & 50 & 40 & 269 \\
\hline PI549.076A/Alpha & 100 & 70 & 50 & 40 & 290 \\
\hline PI549.076A/PI549.076A & 80 & 75 & 70 & 65 & 307 \\
\hline PI194.639/Alpha & 83 & 83 & 80 & 76 & 334 \\
\hline PI194.639/Merit & 87 & 87 & 75 & 73 & 335 \\
\hline PI503.336/PI50.3336 & 89 & 80 & 78 & 77 & 336 \\
\hline PI503.336/Merit & 100 & 100 & 60 & 60 & 340 \\
\hline PI503.336/Alpha & 100 & 95 & 85 & 70 & 365 \\
\hline PI194.639/PI194.639 & 100 & 95 & 90 & 84 & 376 \\
\hline $\operatorname{LSD}(0.05)$ & 35 & 35 & 32 & 27 & 40 \\
\hline
\end{tabular}

a Means are based on 7 to 10 plants per graft combination, and three replications.

${ }^{\mathrm{b}}$ Values based on the following calculation: $\sum^{n-1}{ }_{i=1}\left[\left(X_{i+1}+X_{i}\right) / 2\right]\left[t_{i+1}-t_{i}\right]$, in which $X_{i}=$ percent survival at the $i$ th observation, $t_{i}=$ time (days after inoculation) at the $i$ th observation, and $n=$ total number of observations. At DAI = 1, all plants had 100\% survival (data not shown).
GLM) in SAS (SAS Institute, Cary, NC) with trials and replications treated as random effects and genotypes and grafting combinations as fixed effects. Means were compared with Fisher's protected least significant difference (LSD) values.

\section{RESULTS}

Cotyledon inoculation of single-shoot grafts of cultivars. Disease symptoms appeared on inoculated cotyledons $23 \mathrm{~h}$ after incubation. Infected areas expanded to the node causing water-soaking of the stem and wilting of leaves within 2 days after inoculation (DAI) followed by death of the plants. The experiment $\times$ treatment interaction was not significant, and data from the two trials were combined (Table 1). Based on seven grafting combinations of cross- and self-grafted cultivars, there was no significant difference for plant survival for the first 2 days (Fig. 1). At 3 DAI, a sharp decline in plant survival occurred in all grafts with susceptible shoots; all grafts that used NKS19-90 as shoots had significantly $(P<0.05)$ more plants survive $(90 \%)$, regardless of the genotype used as the rootstock. At 5 DAI, only 5\% of the plants that had susceptible shoots survived, while all grafts that used NKS1990 as shoots had more plants $(P<0.05)$ survive $(40 \%)$.

Cotyledon inoculation of single-shoot grafts of PIs and cultivars. Mean AUSC values for grafts using resistant PI shoots and susceptible cultivar rootstocks (AUSC $=331)$ were greater $(P<0.05)$ than those in reciprocal combinations (AUSC $=183$ ). In self-grafts, plant survival of Alpha and Merit declined more than resistant selfgrafts of PI194.639, PI503.336, and PI549.076A (Table 2). The self-grafts of PI194.639 had greater $(P<0.05)$ AUSC values than PI549.076A, but not greater than PI503.336. At 5 DAI, the percent survivals of Alpha, Merit, PI549.076A, PI194.639, and PI194.639 were, respectively, 9, 20, 65, 77, and 84\%.

Cut stem inoculation of single-shoot grafts. All infected plants had bleached stem symptoms extending down from the point of inoculation. The experiment $\times$ treatment interaction was not significant, and data from the two trials were combined (Table 1). Stem lesion lengths were less after inoculation for grafts with shoots of NKS19-90 than with shoots of Asgrow 2242 and Williams 82 (Table 3). Initially, stem lesion lengths were not different among grafts; however, differences were observed from 10 to 15 DAI. When comparing lesion lengths between grafted resistant and susceptible shoots, resistant shoot grafts were not different, but grafts with susceptible shoots of cv. Williams 82 and Asgrow 2242 had larger $(P$ $<0.05)$ lesions $($ mean $=13.2 \mathrm{~cm}$ ) than resistant shoots $($ mean $=9.2 \mathrm{~cm})$ regardless of the rootstock.

Cut stem inoculation of double-shoot grafts of cultivars. Regardless of the root- 
stock, all susceptible shoots of Williams 82 became infected and died 3 DAI; meanwhile, the resistant shoots of NKS19-90 showed no disease symptoms and survived (Fig. 2A and B). Symptoms on Williams 82 plants began as early as 1 DAI as leaves became flaccid and wilted 2 DAI.

\section{DISCUSSION}

Several studies have demonstrated the association of shoots with physiological traits in soybean when using grafting techniques. For example, hypernodulating mutants were reported to be regulated by factors in plant shoots rather than roots

Table 3. Mean lesion lengths $(\mathrm{cm})$ in cross- and self-grafting combinations using partially resistant (cv. NK S19-90) and susceptible (Asgrow 2242 and Williams 82) soybean genotypes inoculated with Sclerotinia sclerotiorum measured at 7, 9, 11, 13, and 15 days after inoculation (DAI)

\begin{tabular}{lcccrr}
\hline \multirow{2}{*}{$\begin{array}{l}\text { Graft combination } \\
\text { Shoot/root }\end{array}$} & $\mathbf{5}$ & \multicolumn{5}{c}{${\text { Lesion length }(\mathbf{c m})^{\mathbf{a}}}$} \\
\cline { 2 - 6 } & $\mathbf{7}$ & $\mathbf{9}$ & $\mathbf{1 1}$ & $\mathbf{1 3}$ & $\mathbf{1 5}$ \\
\hline NK S19-90/NK S19-90 & 4.4 & 5.7 & 7.1 & 8.6 & 9.6 \\
Asgrow 2242/NK S19-90 & 4.9 & 6.2 & 8.2 & 10.0 & 11.5 \\
Williams 82/NK S19-90 & 5.6 & 7.1 & 9.5 & 11.3 & 13.1 \\
Asgrow 2242/Asgrow 2242 & 6.3 & 7.7 & 10.8 & 13.5 & 15.7 \\
NK S19-90/Asgrow 2242 & 4.1 & 5.0 & 6.5 & 8.0 & 9.0 \\
Williams 82/Williams 82 & 5.4 & 6.7 & 8.9 & 10.8 & 12.6 \\
NK S19-90/Williams 82 & 3.6 & 4.5 & 6.2 & 7.7 & 9.0 \\
LSD (0.05) & 0.9 & 1.0 & 1.3 & 1.5 & 1.9 \\
\hline
\end{tabular}

a Means are based on five plants per graft combination, three replications, and two trials.
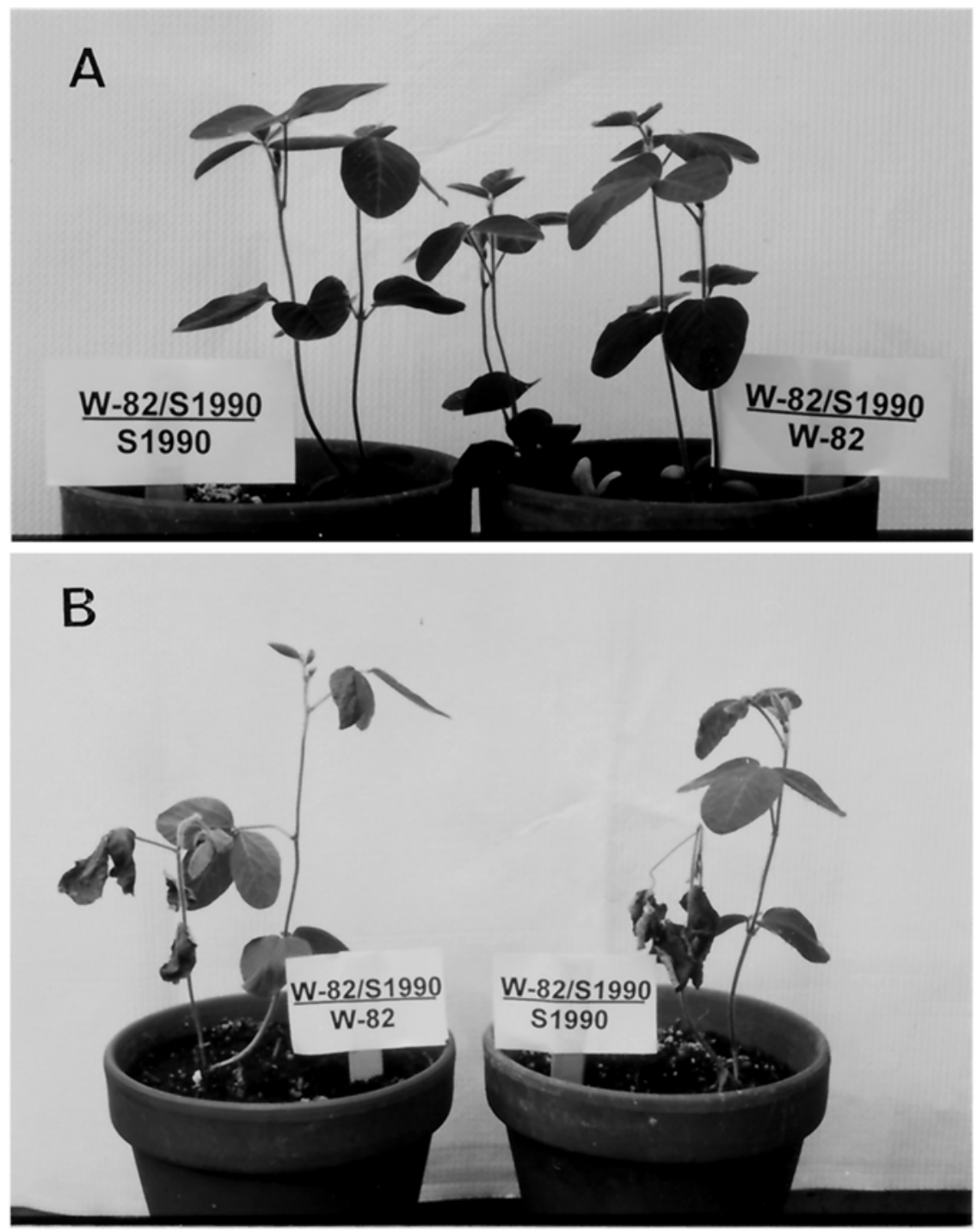

Fig. 2. Response of reciprocal double-shoot grafts made with soybean cvs. Williams 82 (W-82) and NKS19-90 (S19-90) shoots grafted to S19-90 (left) and W-82 (right) rootstocks. A, noninoculated, and $\mathbf{B}$, inoculated with Sclerotinia sclerotiorum.
$(6,7,10)$, as it was shown that autoregulation of nodulation in root systems may involve the presence of shoot-derived inhibitor(s) as well as activator(s) (6). Shoot regulated the decline in the rate of nitrogen fixation (17) due to physiological changes in its components. Factors in shoots of soybean (PI 416.937) primarily controlled salt tolerance (1). However, using similar grafting techniques to study resistance mechanisms of two soilborne soybean diseases, it was concluded that roots played a major role in resistance to sudden death syndrome (18) and brown stem rot (2).

Sclerotinia stem rot of soybeans is primarily an aboveground stem disease, although the fungus is known to infect roots of other crops such as sunflowers (13), and it has been observed to cause basal infection of soybeans as well (G. L. Hartman, data not published). In this study, we evaluated soybean response to $S$. sclerotiorum infection using different shoot and rootstock combinations in either self-, single-, or double-shoot grafts and soybean genotypes. We did not observe in any of these graft combinations, or in noninoculated controls, graft incompatibility related to genotype, and we concluded that the observed plant death was due to the disease. Based on more than 25 graft combinations and two inoculation methods, resistance was greater when the grafted shoot came from a resistant source on a susceptible rootstock compared with the reciprocal combination regardless of the type of grafting technique or inoculation method. In addition, when using double-shoot grafts of one susceptible and one resistant shoot, the susceptible shoot died while the resistant shoot survived regardless of the genotype of the rootstock. Although it seems that shoots are associated with the expression of resistance to $S$. sclerotiorum, there are additional questions related to the role of root resistance in cases where the plant is infected through the roots or the crown.

The different grafting techniques, singleand double-shoot, provided a useful tool to show that $S$. sclerotiorum resistance in soybean originates from shoots and not from roots. More research needs to be conducted to determine the nature of these resistance factors and if they also can be found in roots when the basal portion of the stem or roots is infected. Future studies that investigate the interaction of resistance factors in the plant to oxalic acid produced by the fungus, which has been shown to be important in symptom development in dry beans, soybean, and sunflower $(20,22,23)$, may note that a localized defense response is more likely than a defense product dependent on roots based on our experiments here with different grafting combinations.

\section{LITERATURE CITED}

1. Abd-Alla, M. H., Vuong, T. D., and Harper, J. E. 1998. Genotypic differences in dinitrogen fixation response to $\mathrm{NaCl}$ stress in intact and grafted soybean. Crop Sci. 38:72-77. 
2. Bachman, M. S., and Nickell, C. D. 1999. Use of reciprocal grafting to study brown stem rot resistance in soybean. Phytopathology 89:5963.

3. Bezdicek, D. F., Magee, B. H., and Schillinger, J. A. 1972. Improved reciprocal grafting technique for soybeans (Glycine max L.). Agron. J. 64:558.

4. Bolland, G. J., and Hall, R. 1988. Epidemiology of Sclerotinia stem rot of soybean in Ontario. Phytopathology 78:1241-1245.

5. Chamberlain, D. W. 1951. Sclerotinia stem rot of soybeans. Plant Dis. Rep. 35:490-491.

6. Cho, M. J., and Harper, J. E. 1991. Root isoflavonoid response to grafting between wild-type and nodulation-mutant soybean plants. Plant Physiol. 96:1277-1282.

7. Delves, A. C., Higgins, A. V., and Gresshoff, P. M. 1987. Shoot control of supernodulation in a number of mutant soybeans, Glycine $\max (\mathrm{L})$ Merr. Aust. J. Plant Physiol. 14:689-694.

8. Eathington, S. R., Lim, S. M., Nickell, C. D., Pataky, J. K., and Esgar, R. W. 1993. Disease pressure on soybean in Illinois. Plant Dis. 77:1136-1139.

9. Grau, C. R., Radre, V. L., and Gillespie, F. L. 1982. Resistance of soybean cultivars to Sclerotinia sclerotiorum. Plant Dis. 66:506-508

10. Hamaguchi, H., Kokubun, M., and Akao, S. 1992. Shoot control of nodulation is modified by the root in the supernodulating soybean mutant En6500 and its wild-type parent culti- var Enrei. Soil Sci. Plant Nutr. 38:771-774.

11. Hartman, G. L., Sinclair, J. B., and Rupe, J. C. 1999. Compendium of Soybean Diseases. 4th ed. American Phytopathological Society, St. Paul, MN.

12. Hoffman, D. D., Diers, B. W., Hartman, G. L., Nickell, C. D., Nelson, R. L., Pedersen, W. L., Cober, E. R., Graef, G. L., Steadman, J. R., Grau, C. R., Nelson, B. D., del Rio, L. E., Helms, T., Anderson, T., Poysa, V., Rajcan, I., and Stienstra, W. C. 2002. Selected soybean plant introductions with partial resistance to Sclerotinia sclerotiorum. Plant Dis. 86:971980.

13. Huang, H. C., and Dueck, J. 1980. Wilt of sunflower from infection by mycelialgerminating sclerotia of Sclerotinia sclerotiorum. Can. J. Plant Pathol. 2:47-52.

14. Kim, H. S., and Diers, B. W. 2000. Inheritance of partial resistance to Sclerotinia stem rot. Crop Sci. 40:55-61.

15. Kim, H. S., Hartman, G. L., Manandhar, J. B., Graef, G. L., Steadman, J. R., and Diers, B. W. 2000. Reaction of soybean cultivars to Sclerotinia stem rot in field, greenhouse, and laboratory evaluations. Crop Sci. 40:655-659.

16. Lambert, L., and Kilen, T. C. 1984. Insect resistance factor in soybean PI's 229358 and 227687. Crop Sci. 24:163-165.

17. Malik, N. S. A. 1983. Grafting experiments on the nature of the decline in $\mathrm{N}_{2}$ fixation during fruit development in soybean. Physiol. Plant. 57:561-564
18. Mueller, D. S., Li, S., Hartman, G. L., and Pedersen, W. L. 2000. Evaluation of partial resistance to sudden death syndrome of soybean using reciprocal grafting. (Abstr.) Phytopathology 90:S54.

19. Nelson, B. D., Helms, T. C., and Olson, M. A 1991. Comparison of laboratory and field evaluations of resistance in soybean to Sclerotinia sclerotiorum. Plant Dis. 75:662-665.

20. Noyes, R. D., and Hancock, J. G. 1981. Role of oxalic acid in the Sclerotinia wilt of sunflower. Physiol. Plant Pathol. 18:123-132.

21. Sedun, F. S., and Brown, J. F. 1989. Comparison of three methods to assess resistance in sunflower to basal stem rot caused by Scle rotinia sclerotiorum and $S$. minor. Plant Dis. 73:52-55.

22. Tu, J. C. 1985. Tolerance of white bean (Phaseolus vulgaris) to white mold (Sclerotinia sclerotiorum) associated with tolerance to oxalic acid. Physiol. Plant Pathol. 26:111-117.

23. Wegulo, S. N., Yang, X. B., and Martinson, C. A. 1998. Soybean cultivar responses to Sclerotinia sclerotiorum in field and controlled environment studies. Plant Dis. 82:1264 1270 .

24. Wrather, J. A., Anderson, T. R., Arsyad, D. M., Gai, J., Ploper, L. D., Porta-Puglia, A., Ram, H. H., and Yorinori, J. T. 1997. Soybean disease loss estimates for the top 10 soybean producing countries in 1994. Plant Dis. 81:107-110. 